\title{
Drainage of malignant ascites: patient selection and perspectives
}

\author{
This article was published in the following Dove Press journal: \\ Cancer Management and Research \\ 12 April 2017 \\ Number of times this article has been viewed
}

\section{Maciej Stukan \\ Department of Gynecologic Oncology, Gdynia Oncology Center, Szpitale Wojewodzkie w Gdyni Sp. z o.o., Gdynia, Poland}

Abstract: Malignant ascites (MA) is a sign of advanced cancer and poor prognosis. MA can result in impairment in quality of life (QOL) and significant symptoms. As a supportive treatment, ascites can be drained by paracentesis (PC), percutaneously implanted catheters (tunneled, untunneled, central venous catheters), or peritoneal ports, or peritoneovenous shunts. The aim of this study was to evaluate the effectiveness, safety, and patient-reported outcomes (PRO) of different drainage methods for the management of MA. A systematic review of the literature was performed, and 32 original articles met the inclusion criteria. Patients selected for permanent drain insertion demonstrated symptoms related to MA and had undergone repeated PC. The primary focus of the reviewed articles was procedural safety issues. The rate of technical success of drainage device installation was $100 \%$. Most patients experienced improvements in symptom control after ascites drainage. When analyzed together, $19.7 \%(255 / 1297)$ of patients experienced any complication and 6.2\% (81/1297) experienced serious adverse events during MA drainage. Complications were reported for every drainage method; however, the least occurred after PC or central venous catheter, while the most serious occurred after peritoneovenous shunts. Adverse events were as follows: catheter obstruction: $4.4 \%$, infection: $4.1 \%$, leakage: $3.5 \%$, catheter dislodgment: $2.3 \%$, hypotension: $0.6 \%$, injuries during device insertion: $0.6 \%$, renal impairment: $0.5 \%$, electrolyte imbalance: $0.2 \%$, other: $3.6 \%$. PRO and QOL endpoints were available for 12 studies. When PRO were measured using an interview, a significant improvement in symptom control and QOL was reported in almost all patients. Once standardized questionnaires were used, improvements in symptomatic scores and role functioning were observed. Deterioration was observed in cognitive and emotional subscales. MA drainage is a safe and effective method to control symptoms associated with ascites, and should be perceived as a supportive care, that can be applied for those who need it at any time of their cancer trajectory. Patient selection should be performed using a thorough assessment of symptoms and QOL, and should not be delayed. Keywords: ascites, malignant, cancer, drainage, complications, quality of life, symptoms, palliation, support, catheter, peritoneal port, paracenetesis, shunt, effectiveness, management

\section{Introduction}

Ascites is a common sign of several diseases, both benign and malignant, and often contributes to more symptoms than the underlying pathology itself. ${ }^{1}$ Malignant ascites (MA) is an abnormal accumulation of fluid in the peritoneal cavity as a result of cancer, ${ }^{2}$ and accounts for $\sim 10 \%$ of all cases of ascites. It occurs in many malignancies: adrenal, appendiceal, bladder, breast, cervical, colon, endometrial, esophageal, gall bladder, gastric, liver, lung, lymphoma, melanoma, mesothelioma, neuroendocrine, ovarian, pancreatic, renal, testicular, and thyroid. ${ }^{3-11}$ The most common primary site is epithelial ovarian cancer (EOC), accounting for $38 \%$ of MA occurring in females. ${ }^{12}$ In many
Correspondence: Maciej Stukan Department of Gynecologic Oncology, Gdynia Oncology Center, Szpitale Wojewodzkie w Gdyni Sp. z o.o., Powstania Styczniowego I, 8I-5I9 Gdynia, Poland

$\mathrm{Tel}+48692$ II 248I

$\mathrm{Fax}+48587260296$

Email maciej.stukan@gmail.com 
forms of malignancy, ascites is a sign of advanced disease and poor prognosis, with only $11 \%$ of patients surviving longer than 6 months. EOC is an exception. The majority of women with EOC present with advanced disease (stage III or stage IV) that may include ascites. In these patients, surgical treatment together with combination chemotherapy has resulted in a median progression-free survival of 16-22 months and a 5-year survival rate of $27 \%$, although better results are now being reported with improvements in therapy. ${ }^{13}$

There are two main factors responsible for MA formation. First, obstruction of lymphatic vessels by tumor cells has been suggested as a mechanism for impaired drainage from the peritoneal cavity. Second, vascular endothelial growth factor has been identified as being instrumental in altering the permeability of the peritoneal membrane, inducing angiogenesis and permeabilizing blood vessels, and therefore causing increased filtration into the peritoneal cavity. ${ }^{13}$

The symptoms of MA are abdominal swelling (55\%), abdominal pain (53\%), nausea (37\%), anorexia (36\%), vomiting $(25 \%)$, fatigue (17\%), dyspnea (11\%), early satiety $(6 \%)$, and weight change $(5 \%) .{ }^{13}$

First-line interventions outside the treatment of the primary disease include dietary restriction, diuretics, and repeated large-volume paracentesis (PC). The relative inefficiency of diuretics in specific types of MA is likely because of different pathophysiologic mechanisms for fluid accumulation. ${ }^{14} \mathrm{~A}$ response to diuretics has been observed in patients with ascites secondary to massive hepatic metastases who have a serum:ascitic albumin gradient of $>11 \mathrm{~g} / \mathrm{L} .^{12,15}$

Large-volume PC, where the accumulated fluid is drained intermittently for hours or even days using a fine tube inserted into the abdomen, is widely used to provide short-term relief from the symptoms of MA. ${ }^{16} \mathrm{PC}$ brings immediate but temporary relief in $78 \%-90 \%$ of cases, and implies multiple hospitalizations. ${ }^{2,10,17}$ This approach is undertaken in hospital, as a day-case or inpatient procedure. ${ }^{18}$ Patients often wait until fluid accumulation is substantial to avoid frequent hospital stays, and to ensure the ascites is amenable to drainage, resulting in a deterioration in their quality of life (QOL). ${ }^{16}$

To avoid repeated PC and multiple hospitalizations, a permanently inserted catheter in the abdominal cavity can be considered. Fluid drained from the peritoneal cavity can be directed outside the body, or to other body compartments (eg, venous shunting). In the first case, evacuation of the ascitic fluid can be performed by percutaneous catheters inserted directly through the abdominal wall into the peritoneal cavity (eg, peritoneal, venous catheters), or with a subcutaneous tunnel (eg, PleurX, Tenckhoff). A peritoneal port is a type of tunneled catheter that can be used for ascites evacuation. In this case, there is an intra-abdominal catheter and a port situated in the subcutaneous tissue, which can be easily accessed by repeated puncture.

\section{Rationale}

The management of untreatable ascites, taking into account the limited life expectancy of such patients, must be as minimally invasive and as effective as possible. The ideal treatment should aim to control symptoms and improve QOL, with the least patient discomfort. ${ }^{1}$

Survey studies performed on a cohort of medical professionals have shown variations in the management of MA. There are no definitive data to guide clinicians. ${ }^{19-21}$ Research has focused on the feasibility and complications of management procedures. To date, not much has been published regarding patient-reported outcomes (PRO) in MA drainage. The impetus for this review was to support clinical decision making based on the perspectives of patients, to address uncertainty or variations in practice and possible complications in MA drainage, and to provide a more precise estimate of the effect of MA drainage on patient QOL.

\section{Objectives}

The aim of this systematic review was to evaluate the effectiveness, safety, and PRO of drainage methods for the management of individuals with MA. A secondary aim was to generate a framework to better understand the perceived benefits and barriers that affect individual decision making in offering MA drainage to patients. To this end, the proposed systematic review should answer the following questions:

1. Are there differences between various drainage methods, when comparing feasibility, safety, and efficacy?

2. Are there any specific symptom clusters, QOL issues, or conditions that could direct clinical decision making in MA drainage?

\section{Methods}

A systematic review of the literature concerning feasibility, safety, and the clinical benefit of MA drainage was performed according to the preferred reporting items for systematic review and meta-analysis protocols guidelines, ${ }^{22}$ without protocol registration or amendments.

An initial literature screen, using the keywords "malignant ascites" and "drainage" and "patient perspective" or "quality of life", was performed to determine the dominant study designs. It was found that the majority are retrospective, observational, 
single-arm cohort studies, with $\sim 20-60$ participants. Thus, studies eligible for final analysis were randomized controlled trials, controlled (nonrandomized) clinical trials, all types of original research, prospective and retrospective studies, including case series. Review articles and case reports were excluded from the final analysis, but were consulted for general descriptions of the problem and discussion.

Included studies were those examining patients suffering from MA and any background cancer, and included adults (over 18 years old), both female and male, who underwent drainage of ascites. Excluded studies were those examining patients with non-MA, or describing approaches other than fluid drainage interventions (eg, pharmacology, chemotherapy, biologic agent therapy, cytoreductive surgery, hyperthermia)

Outcomes of interest were safety, feasibility, and efficacy of ascites drainage procedures, PRO, and QOL. Comparators were various methods of ascites drainage. Overall survival was not considered as an outcome because, first, the population of patients with MA is heterogeneous with many different primary cancers contributing to the development of ascites and, second, studies were performed in different settings (clinical oncology, palliative, gynecology, interventional radiology, and ambulatory departments).

MA was defined as an abnormal accumulation of fluid in the peritoneal cavity as a result of cancer. ${ }^{2}$ Ascites drainage was defined as evacuation of the accumulated fluid out of the abdominal cavity. It could be performed by PC, permanently inserted drains, catheters, or shunts, transferring fluid from the abdominal cavity to outside the body or to other body compartments. PC or large-volume PC was defined as a procedure where accumulated fluid was drained intermittently using a fine tube. ${ }^{23}$ Technical success was defined as successful placement of the device and drainage of ascites at completion of the procedure. PRO were defined as measurements that came directly from the patient, both symptoms (one dimensional) and health-related QOL (multidimensional), based on instruments like symptom assessment scales and questionnaires. ${ }^{24}$ If not clearly stated as quantitative data, then descriptive data are provided. Infectious adverse events (AEs) related to catheter placement were graded according to Common Terminology Criteria for Adverse Events (CTCAE v4.0): G2 - localized (local intervention; oral intervention indicated); G3 - intravenous antibiotic, antifungal, or antiviral intervention indicated radiologic or operative intervention indicated; (no G1). Local, peridrain infection was graded $\mathrm{G} 2$, while peritonitis and sepsis were graded G3. Leakage was defined as a flow of ascitic fluid from the peritoneal cavity to outside the body along the catheter, or into subcutaneous tissue.
The included articles were written in English as well as other languages if English language abstracts were provided, which included information that addressed the review objectives.

A literature search was conducted using medical subject headings and words related to MA drainage. PubMed and EBSCO Discovery Service interface (from January 1980 to July 2016) were used to search electronic bibliographic databases such as Medline, Cochrane Library, Web of Science, Academic Search Complete, ScienceDirect, Scopus, Nature Publishing Group, Oxford Journals, Wiley Online Library, and Clinical Key. To ensure literature saturation, a scan of the reference list of included studies or relevant reviews identified though the search was performed.

A Medline search included the following key words: "malignant ascites" and "drainage" AND "patient" and "perspective" or "satisfaction" or "selection" or "quality of life" or "QOL" OR "indication" OR "complication".

The author screened the titles and abstracts yielded by the search against the inclusion criteria. The full reports were obtained for all titles that appeared to meet the inclusion criteria or where there was any uncertainty. The full-text reports were screened and it was decided whether these met the inclusion criteria. The review author was not blinded to the journal titles or the study authors or institutions.

Data that were extracted from studies included: year of publication and clinical setting, study design, number of participants, type of cancer, type of drain/catheter, technical success, complications, PRO, and the quality of the provided data. If these data were not provided, this was recorded or the publication was excluded from the final analysis. Based on these data, the risk of bias was assessed and categorized into low, moderate, or high. The data collected are presented in Table 1 and incorporated into data synthesis in a descriptive way.

Feasibility, safety, and efficacy outcomes of ascites drainage were defined as numbers of patients with technical success of the catheter insertion and the quantity and quality of complications associated with MA drainage.

A systematic narrative synthesis is provided with information presented in the text and tables to summarize and explain the characteristics and findings of the included studies. Results are presented in order by key question and main outcome, followed by additional outcomes. It was originally planned to first report on studies for which the risk of bias was either low or moderate. However, studies with an estimated high risk of bias have been retained for certain key questions or outcomes. 


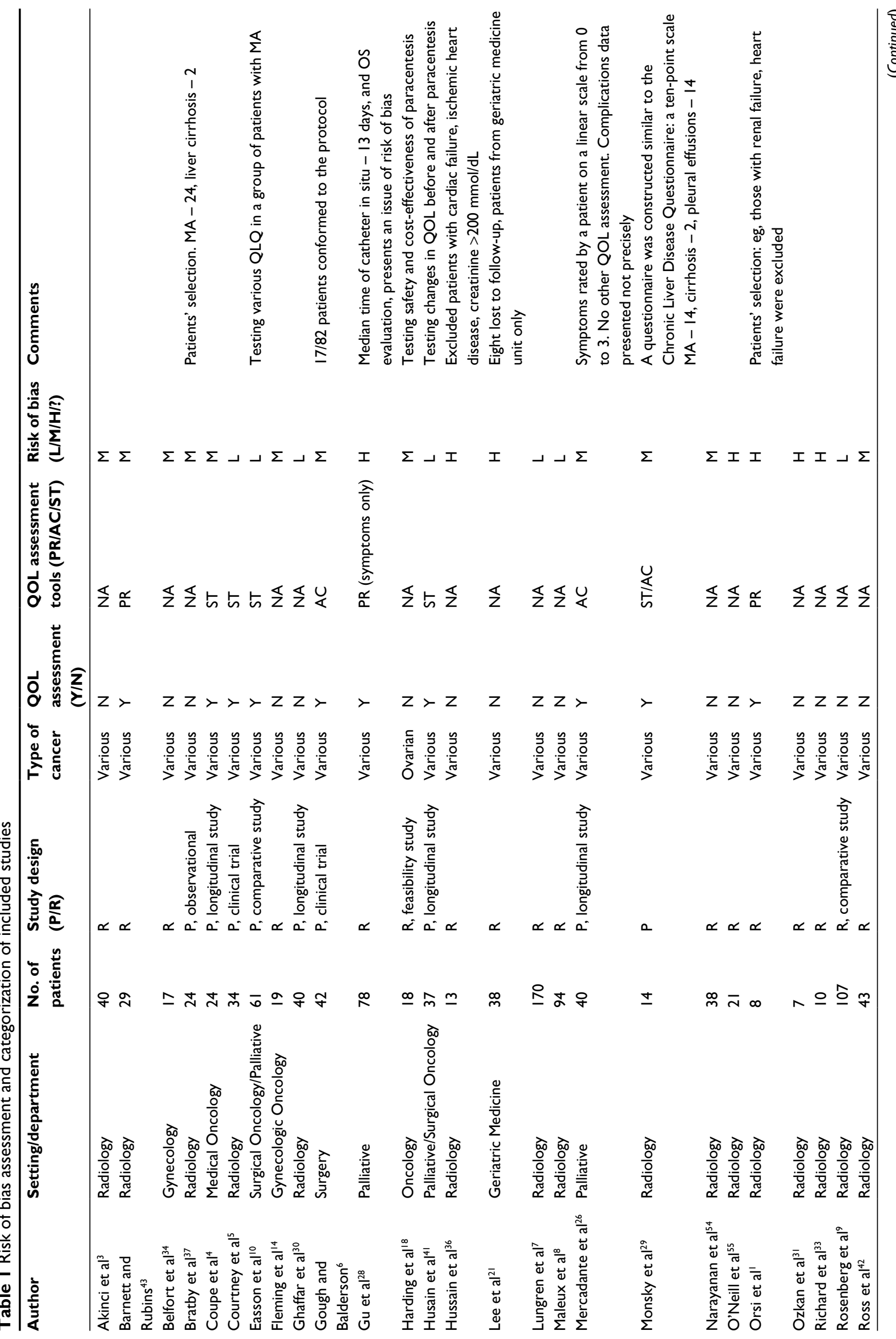




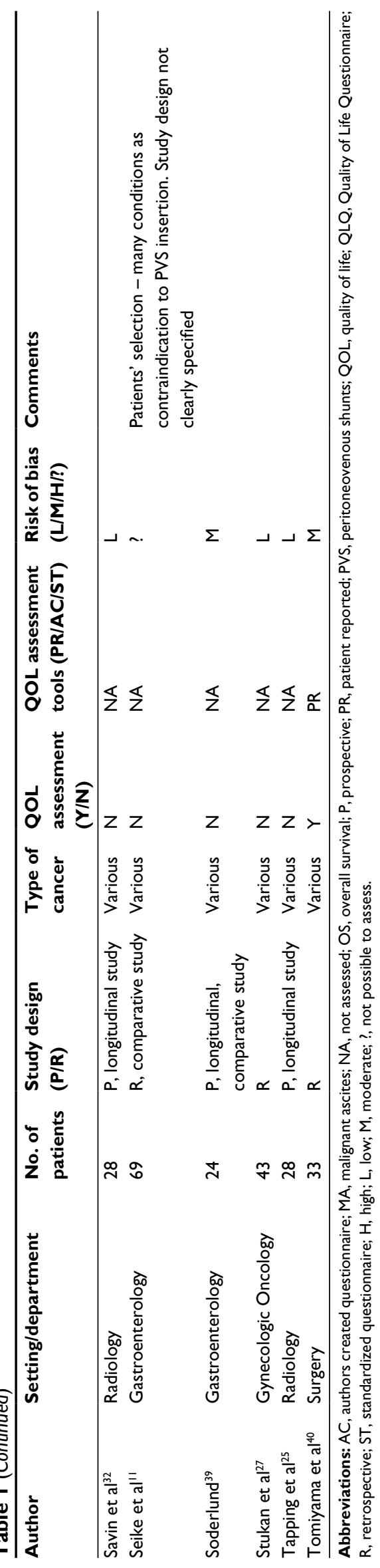

\section{Results}

The literature search revealed 484 articles. Five additional articles were identified from other sources. After screening titles and abstracts, 116 articles were considered relevant. Duplicates $(\mathrm{n}=27)$, review articles $(\mathrm{n}=22)$, and case reports $(n=11)$ were removed. Full-text manuscripts were obtained for all remaining relevant articles or where there was uncertainty. Next, 18 articles were excluded because they were not in line with the objectives of the review (surveys, costeffectiveness analysis, children, hepatic cirrhosis, other). Four articles were excluded because the full texts were not in the English language and the abstracts lacked the required information; one was excluded because no full text or abstract was available; another one was excluded because there was no full text and the abstract did not provide sufficient data. Finally, 32 original articles and case series were included for systematic review.

There were no randomized clinical trials in the included articles. Twelve studies were prospective and 20 were retrospective. The median number of included patients was 33, ranging from 7 to 170 . The risk of bias of the analyzed articles is presented in Table 1.

In most of the studies, patients selected for permanent drain insertion were symptomatic in relation to MA and had undergone repeated PC. The main indication of ascites drainage was symptomatic relief. However, the main focus of the articles was procedural safety issues. Not many studies analyzed real clinical benefits, such as degree of symptom control or QOL. The results are summarized in Table 2 and Table 3.

\section{General considerations}

The rate of technical success of a drainage device installation was $100 \%$. Most of the patients experienced an improvement in symptom control after ascites drainage; however, some authors reported the procedure to be less effective (rate of symptom control: $64 \%-100 \%$ ).

Complications of every drainage method were reported, ranging from $0 \%$ to $67 \%$, with serious adverse events (SAEs) ranging from $0 \%$ to $39 \%$. The lowest level of complications was reported in a group of 18 patients with ovarian cancer and ascites managed using PC $(0 \%)$, followed by PC for various malignancies ( $0 \%-8 \%$, SAE $0 \%-5 \%$ ), drainage using a central venous catheter (CVC; 9\%-25\%, SAE 0\%), permanent peritoneal port (PPP; $4 \%-79 \%$, SAE $0 \%-4 \%$ ), tunneled peritoneal catheters (TPC; $8 \%-56 \%$, SAE $0 \%-39 \%$; for PleurX: $8 \%-56 \%$, SAE $0 \%-12 \%$; for Tenckhoff: $9 \%-29 \%$, SAE $0 \%$ ), and peritoneovenous shunts (PVS; $26 \%-55 \%$, SAE 
Table 2 Malignant ascites drainage - complications' rate and quality, and patients reported outcomes as provided in included literature. Author No. of Type of Technical Symptoms Complications Complications rate (n), by type patients catheter success control rate, all

(\%) (\%)

AE, SAE, Infection Infection Leakage Hypotension Injury

$n(\%) \quad \mathbf{n}(\%) \quad \mathbf{G} 3$

\begin{tabular}{|c|c|c|c|c|c|c|c|c|c|c|c|}
\hline $\begin{array}{l}\text { Akinci et al } \\
(2011)^{3}\end{array}$ & 40 & TPC & $100 \%$ & NA & $13(33 \%)$ & $8(20 \%)$ & 1 & 8 & 0 & 0 & 0 \\
\hline $\begin{array}{l}\text { Barnett, } \\
\text { Rubins } \\
(2002)^{43}\end{array}$ & 29 & TPC & $100 \%$ & $93 \%$ & $5(17 \%)$ & 0 & 1 & 0 & I & 0 & 0 \\
\hline $\begin{array}{l}\text { Belfort et al } \\
(1990)^{34}\end{array}$ & 17 & TPC & $100 \%$ & $100 \%$ & $5(29 \%)$ & $\mathrm{I}(9 \%)$ & 2 & I & 0 & 0 & 0 \\
\hline $\begin{array}{l}\text { Bratby et al } \\
(2007)^{37}\end{array}$ & 24 & PVS & $100 \%$ & NA & II (46\%) & $3(13 \%)$ & 0 & 0 & 0 & 0 & 0 \\
\hline $\begin{array}{l}\text { Coupe et al } \\
(2013)^{4}\end{array}$ & 24 & PPP & $100 \%$ & $100 \%$ & $5(21 \%)$ & $3(13 \%)$ & 0 & 1 & 0 & 1 & 0 \\
\hline $\begin{array}{l}\text { Courtney } \\
\text { et al }(2008)^{5}\end{array}$ & 34 & TPC & $100 \%$ & $83-100 \%$ & $19(56 \%)$ & $4(12 \%)$ & 0 & I & 7 & 0 & I \\
\hline
\end{tabular}

$\begin{array}{llllllllllll}\text { Easson et al } & 61 & \text { PC } & \text { NA } & 78 \% & \text { NA } & \text { NA } & \text { NA } & \text { NA } & \text { NA } & \text { NA } & \text { NA }\end{array}$

$(2007)^{10}$

\begin{tabular}{|c|c|c|c|c|c|c|c|c|c|c|c|}
\hline Fleming & 19 & FP & $100 \%$ & NA & $9(47 \%)$ & $2(11 \%)$ & 0 & I & 2 & 0 & 0 \\
\hline et al & & TPC & & & & & 0 & I & 0 & 0 & 0 \\
\hline$(2009)^{14}$ & & PPP & & & & & 0 & 0 & 0 & 0 & 0 \\
\hline $\begin{array}{l}\text { Ghaffar et al } \\
(2014)^{30}\end{array}$ & 40 & PPP & $100 \%$ & $97.5 \%$ & $2(5 \%)$ & $\mathrm{I}(2.5 \%)$ & 0 & I & I & 0 & 0 \\
\hline $\begin{array}{l}\text { Gough and } \\
\text { Balderson } \\
(1993)^{6}\end{array}$ & 42 & PVS & NA & $64 \%$ & II (26\%) & $2(5 \%)$ & 0 & 0 & NA & 0 & 0 \\
\hline $\begin{array}{l}\text { Gu et al } \\
(2016)^{28}\end{array}$ & 78 & CVC & $100 \%$ & $100 \%$ & $7(9 \%)$ & 0 & 0 & 0 & 7 & 0 & 0 \\
\hline $\begin{array}{l}\text { Harding } \\
\text { et al } \\
(2012)^{18}\end{array}$ & 18 & $\mathrm{PC}$ & $100 \%$ & NA & 0 & 0 & 0 & 0 & 0 & 0 & 0 \\
\hline $\begin{array}{l}\text { Husain et al } \\
(2010)^{41}\end{array}$ & 37 & $P C$ & NA & NA & NA & NA & NA & NA & NA & NA & NA \\
\hline
\end{tabular}




\section{Complications, comments}

\section{QOL}

\begin{tabular}{lll}
\hline $\begin{array}{l}\text { Renal im } \\
\text { pairment imbalance }\end{array}$ & $\begin{array}{l}\text { Electrolyte } \\
\text { imbstruction }\end{array}$ & $\begin{array}{l}\text { Catheter } \\
\text { dislodgement }\end{array}$
\end{tabular}

\section{QOL Results}

assessment

tools

(PR/AC/ST)

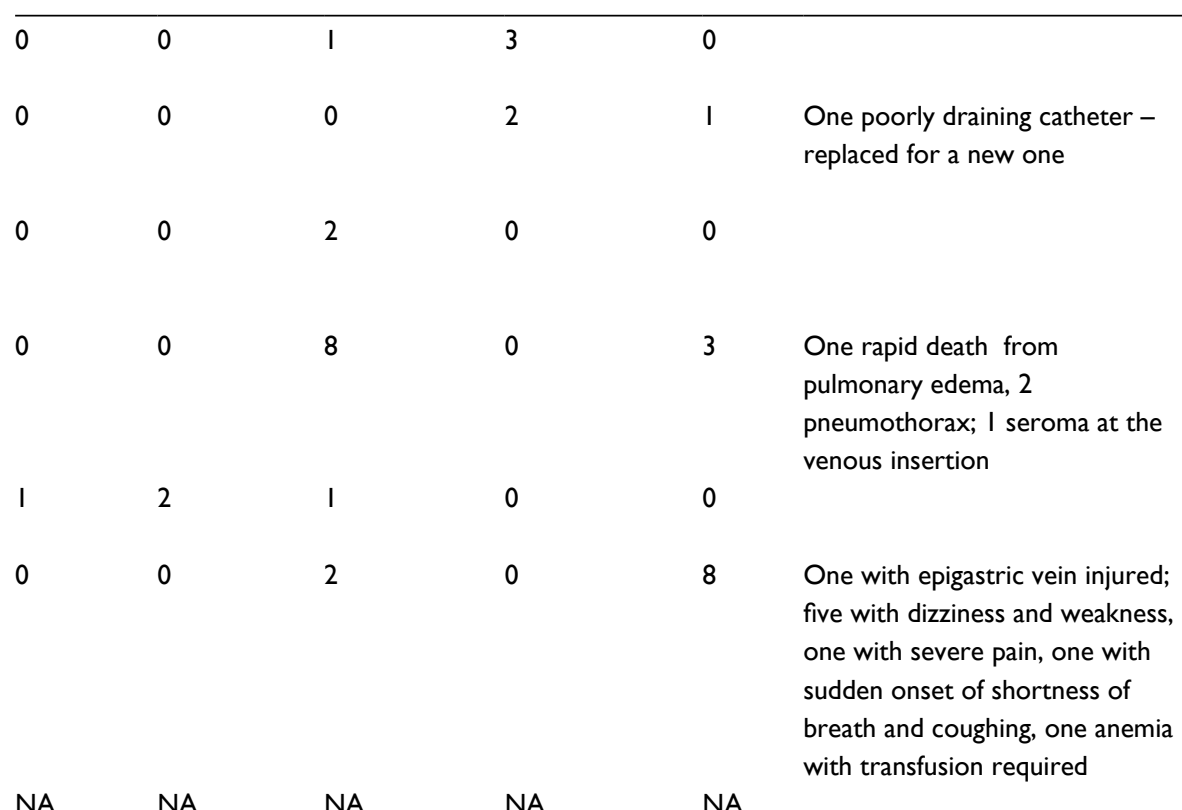

$\begin{array}{llll}\text { NA NA NA } & \text { NA }\end{array}$

0

0

0

0

0

Patients with ovarian cancer only, PC to dryness

wo deaths 6 and 12 hours after AC PVS insertion

NA NA

Self-control, independency.

PR

NA

All patients reported satisfaction and improvement in QOL.

ST (ESAS) Most important: pain and breathlessness relief ${ }^{\text {Ist }}$ week - $56 \%$ reported improved overall QOL, $12^{\text {th }}$ week $-28 \%$ reported improved QOL,

ST: ESAS:AM, Improvement in abdominal EORTC: bloating, anorexia, dyspnea, QLQ-C30, insomnia, fatigue, mobility, QLQ-PAN26 role functioning, general QOL.

Deterioration in cognitive and emotional subscales.

NA NA

0

0

0

2

0

PR

NA

NA

QOL not significant tendency $(P=0.13)$ to improve.

Alleviation in the mean scores for abdominal swelling, anorexia, constipation, fatigue. Comment: mean time of drainage 13 days.

NA

NA NA

NA

NA

NA
ST (ESAS:AM, symptoms of abdominal EORTC distension, shortness of QLQ-C30) breath, role functioning. Global QOL did not improve. The domain of cognitive functioning declined, emotional - trend toward decline. 
Table 2 (Continued)

\begin{tabular}{|c|c|c|c|c|c|c|c|c|c|c|c|}
\hline \multirow[t]{2}{*}{ Author } & \multirow[t]{2}{*}{$\begin{array}{l}\text { No. of } \\
\text { patients }\end{array}$} & \multirow[t]{2}{*}{$\begin{array}{l}\text { Type of } \\
\text { catheter }\end{array}$} & \multirow{2}{*}{$\begin{array}{l}\text { Technical } \\
\text { success } \\
(\%)\end{array}$} & \multirow{2}{*}{$\begin{array}{l}\text { Symptoms } \\
\text { control } \\
\text { (\%) }\end{array}$} & \multicolumn{2}{|c|}{$\begin{array}{l}\text { Complications } \\
\text { rate, all }\end{array}$} & \multicolumn{5}{|c|}{ Complications rate $(n)$, by type } \\
\hline & & & & & $\begin{array}{l}\mathrm{AE}, \\
\mathrm{n}(\%)\end{array}$ & $\begin{array}{l}\text { SAE, } \\
\text { n (\%) }\end{array}$ & $\begin{array}{l}\text { Infection } \\
\text { G2 }\end{array}$ & $\begin{array}{l}\text { Infection } \\
\text { G3 }\end{array}$ & Leakage & Hypotension & Injury \\
\hline $\begin{array}{l}\text { Hussain } \\
\text { et al } \\
(2004)^{36}\end{array}$ & 13 & PVS & $100 \%$ & $92 \%$ & $5(38 \%)$ & $\mathrm{I}(8 \%)$ & 0 & 0 & 0 & 0 & 0 \\
\hline $\begin{array}{l}\text { Lee et al } \\
(2000)^{21}\end{array}$ & 38 & TPC & $100 \%$ & $100 \%$ & $20(53 \%)$ & $15(39 \%)$ & 0 & 13 & 0 & 2 & 0 \\
\hline $\begin{array}{l}\text { Lungren } \\
\text { et al }(2013)^{7}\end{array}$ & 170 & TPC & $100 \%$ & NA & $14(8 \%)$ & $2(1 \%)$ & 3 & 2 & 4 & 0 & 0 \\
\hline $\begin{array}{l}\text { Maleux et al } \\
(2016)^{8}\end{array}$ & 97 & TPC & $100 \%$ & NA & $9(9 \%)$ & 0 & 2 & 0 & 4 & 0 & 0 \\
\hline $\begin{array}{l}\text { Mercadante } \\
\text { et al } \\
(2008)^{26}\end{array}$ & 40 & CVC & $100 \%$ & $75 \%$ & $10(25 \%)$ & 0 & 0 & 0 & 6 & 0 & 0 \\
\hline $\begin{array}{l}\text { Monsky } \\
\text { et al } \\
(2009)^{29}\end{array}$ & 14 & PPP & $100 \%$ & $96 \%$ & II (79\%) & 0 & 0 & 0 & 4 & 0 & 3 \\
\hline $\begin{array}{l}\text { Narayanan } \\
\text { et al } \\
(20 \mid 4)^{54}\end{array}$ & 38 & TPC & $100 \%$ & NA & $9(24 \%)$ & $2(5 \%)$ & 0 & 2 & 2 & 0 & 0 \\
\hline $\begin{array}{l}\text { O'Neill et al } \\
(200 I)^{55}\end{array}$ & 24 & TPC & $100 \%$ & $100 \%$ & $4(17 \%)$ & $3(12 \%)$ & 0 & 3 & 0 & 0 & 0 \\
\hline $\begin{array}{l}\text { Orsi et al } \\
(2002)^{\prime}\end{array}$ & 8 & PVS & $100 \%$ & $100 \%$ & 0 & 0 & 0 & 0 & 0 & 0 & 0 \\
\hline $\begin{array}{l}\text { Ozkan et al } \\
(2007)^{3 !}\end{array}$ & 7 & PPP & $100 \%$ & NA & $4(57 \%)$ & 0 & 0 & 0 & 2 & 0 & 0 \\
\hline $\begin{array}{l}\text { Richard } \\
\text { et al } \\
(2001)^{33}\end{array}$ & 10 & TPC & $100 \%$ & NA & $2(20 \%)$ & 0 & 0 & 0 & 0 & 0 & 0 \\
\hline Rosenberg & 40 & TPC & $100 \%$ & NA & $3(8 \%)$ & $\mathrm{I}(3 \%)$ & 0 & I & I & 0 & 0 \\
\hline et al $(2004)^{9}$ & 67 & PC & $100 \%$ & & $5(8 \%)$ & $3(3 \%)$ & 0 & 3 & 0 & 0 & 0 \\
\hline $\begin{array}{l}\text { Ross et al } \\
(1989)^{42}\end{array}$ & 43 & PC & $100 \%$ & $87 \%$ & $3(7 \%)$ & $2(5 \%)$ & 0 & 0 & 0 & 3 & 0 \\
\hline $\begin{array}{l}\text { Savin et al } \\
(2005)^{32}\end{array}$ & 28 & PPP & $100 \%$ & $96 \%$ & $\mathrm{I}(4 \%)$ & I(4\%) & 0 & I & I & 0 & 0 \\
\hline Seike et al & 20 & PVS & $100 \%$ & NA & II (55\%) & $3(15 \%)$ & I & 0 & 0 & 0 & 3 \\
\hline$(2007)^{\prime \prime}$ & 49 & PC & & & $7(14 \%)$ & $7(14 \%)$ & 0 & I & 0 & 1 & 0 \\
\hline $\begin{array}{l}\text { Soderlund } \\
(1986)^{39}\end{array}$ & 24 & PVS & NA & NA & $16(67 \%)$ & $6(25 \%)$ & 0 & 0 & 0 & 0 & 0 \\
\hline $\begin{array}{l}\text { Stukan et al } \\
(2015)^{27}\end{array}$ & 43 & CVC & $100 \%$ & $100 \%$ & $5(12 \%)$ & 0 & I & 0 & 0 & 1 & 0 \\
\hline
\end{tabular}




\section{Complications, comments}

\section{QOL}

\begin{tabular}{lll}
\hline $\begin{array}{l}\text { Renal im } \\
\text { pairment imbalance }\end{array}$ & $\begin{array}{l}\text { Electrolyte } \\
\text { imbstruction }\end{array}$ & $\begin{array}{l}\text { Catheter } \\
\text { dislodgement }\end{array}$
\end{tabular}

QOL

assessment

tools

(PR/AC/ST)

0

2

5

$\begin{array}{llll}0 & 0 & 5 & 0 \\ 0 & 0 & 0 & 5 \\ 0 & 0 & 1 & 1 \\ 0 & 0 & 0 & 4 \\ 0 & 0 & 2 & 0\end{array}$

0

0

0

0

0

0

0

0

0

0

2

0

0

I

I

0
0
0

0
5

0
0

4

0

0

0

7

2

3

2
One procedure-related
mortality - pulmonary edema

NA

Two with fatal hypotension;

eight were lost to follow-up;

0

NA

NA

NA NA

NA NA

Catheter "not working" in 17 AC cases - not precisely explained, including dislodgment.

2

Three hematoma at port

ST/AC reservoir; four leakage to subcutaneous tissue / port site; two port site metastasis

Other: three patients reported pain, one sleep disturbances.

NA

NA

Patients' selection: e.g. those

PR renal failure, heart failure were excluded.

$0 \quad$ Long-term patency $100 \%$, but each time flushed with heparine.

NA None of minor complications affected drainage.

0

NA

ascites loculations : PC $-2, \quad$ NA

TPC - I.

Two fatal procedure-related NA hypotension

0 One patient experiences leakage NA and subsequently peritonitis; 22/28 had MA

3 PVS: one DIC; one fatal pulmonary embolism, one pulmonary edema; three subcutaneous bleeding; PC: all SAE were considered procedure-related

6 One fatal pulmonary edema related to the procedure; five thromboembolisms

One patient with an occult primary and PS4 experienced hypotension, renal impairment and electrolyte imbalance
Results

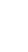


Table 2 (Continued)

\begin{tabular}{|c|c|c|c|c|c|c|c|c|c|c|c|}
\hline \multirow[t]{2}{*}{ Author } & \multirow[t]{2}{*}{$\begin{array}{l}\text { No. of } \\
\text { patients }\end{array}$} & \multirow[t]{2}{*}{$\begin{array}{l}\text { Type of } \\
\text { catheter }\end{array}$} & \multirow{2}{*}{$\begin{array}{l}\text { Technical } \\
\text { success } \\
(\%)\end{array}$} & \multirow{2}{*}{$\begin{array}{l}\text { Symptoms } \\
\text { control } \\
\text { (\%) }\end{array}$} & \multicolumn{2}{|c|}{$\begin{array}{l}\text { Complications } \\
\text { rate, all }\end{array}$} & \multicolumn{5}{|c|}{ Complications rate $(n)$, by type } \\
\hline & & & & & $\begin{array}{l}\mathrm{AE}, \\
\text { n (\%) }\end{array}$ & $\begin{array}{l}\text { SAE, } \\
\text { n (\%) }\end{array}$ & $\begin{array}{l}\text { Infection } \\
\text { G2 }\end{array}$ & $\begin{array}{l}\text { Infection } \\
\text { G3 }\end{array}$ & Leakage & Hypotension & Injury \\
\hline $\begin{array}{l}\text { Tapping et al } \\
(2012)^{25}\end{array}$ & 28 & TPC & $100 \%$ & NA & II (39\%) & 0 & 2 & 0 & 3 & 0 & 0 \\
\hline $\begin{array}{l}\text { Tomiyama } \\
\text { et al } \\
(2006)^{40}\end{array}$ & 33 & PVS & $100 \%$ & NA & $18(55 \%)$ & II (33\%) & 0 & 0 & 0 & 0 & 1 \\
\hline
\end{tabular}

Notes: Technical success: successful catheter placement; injury: bowel, intra-abdominal, or abdominal wall vessels injury; GI-3: grading of AEs according to CTCAE (catheter-related infection); G2: localized; local intervention, indicated; oral intervention, indicated; G3: IV antibiotic, antifungal, or antiviral intervention indicated; radiologic or operative intervention indicated.

Abbreviations: AC, authors created questionnaire; AE, adverse event; CTCAE, Common Terminology Criteria for Adverse Events; CVC, central venous catheter (inserted intra-abdominally for ascites drainage); DIC, disseminated intravascular coagulation; EORTC, European Organization for the Research and Treatment of Cancer; ESAS, Edmonton Symptom Assessment Scale; ESAS:AM, ESAS - Ascites Modification; FP, French pigtail; IV, intravenous; MA, malignant ascites; MSAS, Memorial Symptom Assessment Survey; NA, not assessed/not applicable; PC, paracentesis; PPP, permanent peritoneal port; PR, patient reported; PVS, peritoneovenous shunts; QOL, quality of life; QLQ-C30, Quality of Life Questionnaire Core; QLQ-PAN26, Quality of Life Questionnaire Pancreatic cancer module; SAE, serious adverse event; SSQ, subjective significance questionnaire; ST, standardized questionnaire; TPC, tunneled peritoneal catheters (PleurX, Tenckhoff, other peritoneal)

$5 \%-33 \%)$. The most serious procedure-related complications were reported for PVS (mortality from pulmonary edema and thromboembolism).

When analyzed together, $19.7 \%(255 / 1297)$ of patients experienced any complication and 6.2\% (81/1297) experienced an SAE during MA drainage. Generally, the percentage of complications was as follows: infection, G2 - 1\%; infection, G3 $-3.1 \%$; leakage $-3.5 \%$; hypotension $-0.6 \%$; injuries during device insertion $-0.6 \%$; renal impairment $0.5 \%$; electrolyte imbalance $-0.2 \%$; catheter obstruction $4.4 \%$; catheter dislodgment $-2.3 \%$; other $-3.6 \%$. Detailed data for various drainage methods are presented in Table 3.

\section{TPC}

In the study with the largest number of patients, $170 \mathrm{TPC}$ were inserted for the treatment of MA, with a $100 \%$ technical success rate for catheter insertion and no procedure-related deaths or major placement complications. Fourteen $(8 \%)$ post-placement complications were identified. Five patients experienced catheter malfunction. Four patients suffered leakage of ascites that occurred at an average of 11.25 days after catheter placement at the incisional site, which required suture placement around the tunnel. Three demonstrated cellulitis of the tunnel tract and two developed peritonitis. The annual complication event rate was 0.43 events per year (i.e., 0.12 events per 100 catheter-days). ${ }^{7}$ Pancreatic cancer was associated with a statistically greater catheter complication rate ( $P=0.007$; odds ratio, $4.7 ; 95 \%$ confidence interval, 1.399-15.511). These included four catheter occlusions that were addressed by catheter replacement and one case of cellulitis treated successfully using antibiotic therapy. ${ }^{7}$
Based on a 28-patient study, the factors significantly associated with complications of ascites drainage were as follows: current chemotherapy, low albumin levels, high white blood cell count, and renal dysfunction. ${ }^{25}$ In contrast, in a study of 170 patients, the authors found none of these factors to be significantly associated with catheter complications. ${ }^{7}$ In a prospective clinical trial, no significant changes in sodium, potassium, creatinine, albumin, or total protein were detected when compared to levels at baseline and 12 weeks of drainage. ${ }^{5}$ When Tenckhoff TPC were used for MA drainage $(n=94)$, an analysis suggested that patients with widespread gastrointestinal cancers and refractory MA had a higher risk for early death, compared with the reference group of patients with widespread metastatic gynecologic cancers. Patients with pancreatic cancer $(n=11)$ were analyzed in a separate hepatobiliary cancer group $(n=22) .^{8}$

\section{CVC}

CVC was used to drain ascites. The technique was not painful and was easily accepted by patients. Insertion was technically successful in all patients, but one who required a second attempt. A mean admission time of 5.5 days (range 2-14 days) was reported, and the mean drained volume during admission was $8499 \mathrm{~mL}$ (range 800-20700 mL), of which the mean was $2850 \mathrm{~mL}(300-4200 \mathrm{~mL})$ in the first 24 h. ${ }^{26}$ The CVC could also be inserted, with local anesthesia, under ultrasound guidance, with $100 \%$ technical success, without procedure-related complications, in an outpatient department. ${ }^{27}$ In the case of CVC usage, no significant infection or insertion-related AEs were reported. Mechanical problems were the main issue, and included leakage, 


\begin{tabular}{|c|c|c|c|c|c|c|c|}
\hline & & & & & Complications, comments & QOL & \\
\hline $\begin{array}{l}\text { Renal im } \\
\text { pairment }\end{array}$ & $\begin{array}{l}\text { Electrolyte } \\
\text { imbalance }\end{array}$ & Obstruction & $\begin{array}{l}\text { Catheter } \\
\text { dislodgement }\end{array}$ & Other & & $\begin{array}{l}\text { QOL } \\
\text { assessment } \\
\text { tools } \\
\text { (PR/AC/ST) }\end{array}$ & Results \\
\hline 0 & 0 & 0 & 4 & 2 & One incisional site hernia & NA & \\
\hline 0 & 0 & 4 & 2 & 11 & $\begin{array}{l}\text { Eight developed DIC - one died } \\
\text { from DIC; three pulmonary } \\
\text { edema - one died; one wound } \\
\text { hematoma }\end{array}$ & PR & $\begin{array}{l}60 \% \text { of patients reported } \\
\mathrm{QOL} \text { improvement }\end{array}$ \\
\hline
\end{tabular}

catheter dislodgment, or "not working". These were managed using local procedures such as additional sutures, use of a bag to collect the fluid if leakage occurred, and catheter replacement. ${ }^{26-28} \mathrm{CVCs}$ are rather ineffective in patients with mucinous ascites. ${ }^{27}$ Patients reported alleviation of all the ascites-related symptoms after drainage by CVC compared with baseline, with abdominal swelling, anorexia, and constipation being the most significantly changed (the severity of the symptoms was assessed using a linear scale). ${ }^{28}$ In another study, it was the impression of the health care team that patients were very comfortable following CVC placement and reported better symptom control and an increased intake of food and nutritional supplements. ${ }^{27}$

\section{PPP}

PPP offers a convenient and relatively safe alternative to frequent $\mathrm{PC}$ in the management of refractory ascites, and results in symptomatic improvement for most patients. Ports were inserted late in the disease trajectory in patients who were heavily pretreated using chemotherapy. Infection occurred in $0 \%-4 \%$ of patients and was easily treated. Most catheter-related morbidity occurred early after placement, was self-limited, and did not affect ascites drainage. ${ }^{4,29-31}$ In a study testing PPP usage for ascites drainage, 1 of 24 patients suffered from grade 3 hypotension. That patient had $9.3 \mathrm{~L}$ of ascites drained on that occasion, and therefore, the complications were likely secondary to excessive ascitic fluid removal and not directly because of the peritoneal port. ${ }^{4}$ Leakage to subcutaneous tissue was reported in 4\%-29\% of patients after PPP insertion for ascites drainage. ${ }^{29,31,32}$ Hypoalbuminemia was a common adverse effect, but it was assumed that it was most likely influenced by disease progression, and was therefore not entirely attributable to ascitic drainage. ${ }^{4}$ No significant protein loss was observed in patients when a high-protein diet was combined with frequent, small volume removal of ascites. . $^{9,33,34}$
MA drainage using PPP can improve the QOL for patients with end-stage disease. On a ten-point scale, QOL improvement, compared with that prior to port placement, was rated a mean of 9.5 by the patients and 9.0 by the nursing staff. The 1-week QOL was lowest, with a mean of 8.7, compared with the 1- and 3-month intervals, which were similar. Both patients and nurses reported a high degree of convenience (rated at 9.7 and 9.6, respectively) and improvement of symptoms and comfort (9.6 and 9.3, respectively). Patients commonly stated that they were significantly more mobile and better able to perform daily activities, including more strenuous activities such as gardening. Seven patients (7/14) stated that they were able to travel for important family obligations and vacations, with minor arrangements being made for port aspiration at local hospitals by nursing staff or family. ${ }^{29}$ Thirty-nine $(97.5 \%)$ patients were treated successfully without catheter manipulation, or antibiotic therapy, and showed complete relief of symptoms and good compliance until death. Avoidance of repeated PC was satisfactory to patients and clinicians. ${ }^{30}$ Subcutaneous tumor growth was observed along the tunneled catheter in two patients, 4 and 6 months after placement. These patients reported associated mild discomfort and palpable masses. External beam irradiation was considered for palliation. ${ }^{29}$

\section{PVS}

PVS transfer fluid from the peritoneal cavity to the systemic circulation through a one-way compressible valve system. Fluid is maintained within the body, minimizing protein and electrolyte loss, and reducing patient discomfort associated with repeated PC. ${ }^{35}$ This technique permits home management, but requires careful patient selection and post-procedure management to avoid serious complications from fluid overload. ${ }^{6,36}$ The most common cause of catheter dysfunction was fibrin sheath formation at the venous limb 
in a significant number of patients. Successful revision of the shunt could be achieved in the majority. ${ }^{6,37}$ Another reported complication after PVS insertion was pneumothorax, which occurred more commonly with subclavian puncture compared with other sites of venous access. ${ }^{37,38}$

Twenty patients underwent PVS placement and 49 patients were subjected to PC. After PVS, abdominal girth decreased significantly. The median number of procedures was less in the PVS group than in the PC group (one vs two, respectively; $P<0.0001)$. The postoperative performance score (PS) was significantly improved following PVS placement $(P=0.0026)$. Severe complications were observed in one patient in the PVS group and in seven patients in the PC group. ${ }^{11}$

The authors of a prospective study reported considerable morbidity $(41 \%)$ and mortality $(6 \%$ - procedure-related pulmonary edema and sepsis) after PVS insertion, but also reported its efficiency in certain cases. ${ }^{39}$ In a consecutive cohort of patients who underwent PVS insertion, as many as $55 \%$ experienced complications, $33 \%$ were serious, and two patients died (from disseminated intravascular coagulation and pulmonary edema). No distant metastasis was found..$^{40}$ In another study, a $21 \%$ risk of thromboembolism events was reported. ${ }^{39}$ However, with the use of low-dose warfarin, disseminated intravascular coagulation was not seen after PVS insertion. ${ }^{37}$ Interestingly, in a series of 24 patients with MA and 30 with ascites secondary to liver cirrhosis, managed using PVS insertion, septic complications were observed in the cirrhotic patient group only ${ }^{39}$ Patients with PS of 40-50 (Karnofsky score) before PVS insertion experienced significantly less improvement after the procedure, compared with those with PS of 60 or higher. ${ }^{11}$ The importance of careful patient selection for the PVS procedure was emphasized.

\section{$\mathrm{PRO}$ and $\mathrm{QOL}$ issues}

PRO and QOL endpoints were available in 12 of 32 evaluated studies. They were reported as general patient impressions, simple linear scales for evaluation of symptoms, or standardized questionnaires, sometimes modified for specific clinical situations.

Based on studies that provided data, symptom control was achieved in a median of $97 \%$ of patients after MA drainage: $78 \%-100 \%$ for PC, $83 \%-100 \%$ for TPC, $75 \%-100 \%$ for CVC, and $96 \%-100 \%$ for PPP.

When PRO were measured using an interview or a health care impression, a significant improvement in symptom control and QOL was reported in almost all patients, with the exception 
of one study (using PVS), where only $60 \%$ of patients claimed an improvement. However, once standardized questionnaires were used, before and after MA drainage, improvements in symptomatic scores associated with ascites (abdominal distension, shortness of breath, anorexia, insomnia, fatigue, and mobility) and role functioning were observed, whereas deterioration was seen in the emotional and cognitive subscales; $5,10,41$ general QOL improved ${ }^{10}$ or did not change. ${ }^{41}$ Moreover, the symptom clusters found in patients with MA suggest that they have patterns of symptoms characteristic of patients with advanced disease, not just symptoms commonly associated with ascites. ${ }^{41}$ In $25 \%$ of patients admitted to a palliative care unit, no relevant changes in symptom burden were observed, despite effective removal of a large amount of abdominal fluids. ${ }^{26}$ The patient-reported change in symptoms did neither correlate with the amount of fluid drained nor with any baseline symptom scores. If pain scores were high at baseline, patients were not greatly relieved after MA drainage. A worse baseline PS (50\% of patients had PS 3-4 Eastern Cooperative Oncology Group [ECOG] score) was predictive of a better patient-reported change in symptoms. ${ }^{10}$ After single PC, the duration of symptomatic relief ranged from 4 to 45 days (mean 10.4 days). ${ }^{42}$

Two clusters of symptoms common at both pre- and postPC time points were found: depression-anxiety and fatiguemobility-well-being-appetite. ${ }^{41}$ When considering testing the QOL of patients using questionnaires, it was noted that, clinically, those in whom "well-being" clusters with "fatigue" and "mobility" may be more likely to benefit from PC and, therefore, more likely to be able to complete and return the post-PC questionnaires. While those in whom "well-being" migrates to the anxiety-depression cluster, symptoms which do not improve significantly with PC, might be less likely to complete and return the post-PC questionnaires. PC does not seem to target the symptoms found to cluster but rather single symptoms, without significant gains in many of the well-being and QOL domains. ${ }^{41}$ According to Husain et al, symptom cluster methodology can contribute to the evidence for PC and other procedures in MA by identifying patients, grouped by their cluster profiles, who may respond differently to a procedure. However, they acknowledged that a study with larger sample size would be required to test the concept. Using symptom cluster methods also allows to consider alternate interventions that target the constellation of symptoms that cluster in these patients to realize gains in well-being and QOL. ${ }^{41}$

Avoidance of repeated $\mathrm{PC}$ and repeated admission to hospital was satisfactory to patients and clinicians after placement of $\mathrm{PPP}^{30}$ or PVS. ${ }^{6}$ In a series of 17 patients who underwent insertion of a peritoneal drain (published in 1990), all reported satisfaction with the drain and felt that their lifestyle was significantly improved by the device. ${ }^{34}$ Patients with permanently inserted catheters can control ascites drainage by themselves, and therefore are not confined to the area of their hospital for repeated PC. ${ }^{27,43}$

\section{Discussion}

This systematic review of the literature about various drainage methods for the management of individuals with MA showed almost $100 \%$ feasibility of all available methods, including PC, TPC, CVC, PPP, and PVS. There were differences in safety issues, with the lowest rate of $5.5 \%$ of $\mathrm{AE}$ for PC and $0 \%$ of SAE for CVC. The latest had a $13.7 \%$ rate of minor complications. Comparable safety profile was found for TPC and PPP with the AE rate of $20.3 \%$ and $20.0 \%$ and SAE of $6.5 \%$ and $4.3 \%$, respectively. Special attention and experience are crucial for selecting patients for PVS because AE and SAE rates were $43.9 \%$ and $15.9 \%$, respectively. Efficacy defined with symptom control was achieved in 64\%-100\%, generally for all methods. Patients with MA who benefit from drainage the most are those who present with symptoms associated with ascites (abdominal distension, dyspnea, anorexia, insomnia, fatigue, and mobility difficulties). Other QOL issues such as role, emotional, and cognitive functioning should also be considered. The underlying malignancy, its symptoms, and prognosis are important to direct clinical decision making in MA drainage. The life expectancy of patients with refractory MA is very poor, with an overall survival ranging from 1 to 6 months in nonovarian cancer and 10-24 months in ovarian cancer. ${ }^{8,12,44}$ Patients with refractory MA with a longer life expectancy, such as patients with gynecologic tumors, may also benefit from the use of a tunneled catheter for a longer period, compared with patients with more aggressive tumors such as gastrointestinal malignancies. ${ }^{8}$

According to survey studies, the most commonly used procedure for MA management was PC. Up to $48 \%$ of physicians prescribed diuretics. ${ }^{19,20}$ In Germany and Austria, one-third of responders who specialized in gynecology and gastroenterology preferred catheter drainage over PC; very few medical oncologists chose the application of a catheter. ${ }^{20}$ In the UK, about half of responders had never used permanent indwelling catheters. ${ }^{19}$

Findings from multiple studies indicate that integrating palliative care early in the disease trajectory can result in improvements in QOL, symptom control, patient and caregiver satisfaction, illness understanding, quality of end-of-life 
care, survival, and costs of care. ${ }^{45,46}$ When a patient with MA presents for PC, this should be a flag to signal the need to initiate a comprehensive management plan that includes appropriate referral to supportive and psychosocial care. ${ }^{41}$

Assessment of patients with MA should include measurement of the more global symptoms of fatigue, well-being, depression, and anxiety, in addition to shortness of breath, abdominal distension, and mobility. It has been suggested that examining the benefits and potential side effects of PC should be performed using a thorough assessment of symptoms and QOL. ${ }^{41}$ The European Organization for the Research and Treatment of Cancer Quality of Life Questionnaire (EORTC QLQ-C30) and the Edmonton Symptom Assessment ScaleAscites Modification together, or the EORTC QLQ-C30 with the addition of the Quality of Life Questionnaire, pancreatic cancer module (QLQ-PAN26) ascites and abdominal pain subscales could be used. ${ }^{10}$

Altogether, the successful management of MA using intraperitoneal catheters is most likely multifactorial and dependent on underlying malignancy, catheter type, procedure, operator experience and setting, and the PS of the patient, comorbidities, nutritional status, and the ability to care for the device. Insertion of TPC, PPP, or PVS involves many medical resources. ${ }^{5-7,25,30,37}$ For patients with EOC, a PC to dryness can be safely performed as an outpatient, but takes 6-8 h. ${ }^{18}$ In contrast, insertion of a CVC into the abdominal cavity for ascites drainage is easy, and can be performed in an outpatient department, under local anesthesia, using a short ultrasound assessment to determine the safest place to position the catheter. ${ }^{27}$ Moreover, the CVC provides all the advantages of a permanent drain.

There are many benefits to permanently inserted catheters (TPC, CVC, PPP) for MA management. Patients can drain their ascites independently of health care staff or facilities, at home, at times that they need to control symptoms, and to a reasonable degree (not to dryness, as often performed at hospitals), so that many complications can be prevented (eg, hypotension, injury, leakage, rapid protein loss, or electrolyte imbalance). All these factors contribute to better symptom control and QOL. Family caregivers benefit as well. There are some risks associated with permanent catheter usage that should be addressed. The most important is infection, and others are fluid leakage, drain obstruction, or dislodgment.

Systemic chemotherapy did not increase the risk of catheter-related infection after TPC or CVC placement for MA. Therefore, chemotherapy should not be a contraindication to catheter placement. ${ }^{27,47}$ An additional advantage of TPC or PPP for ascites drainage is that intraperitoneal infusion of chemotherapy (cisplatin) or active targeted therapeutic agents (catumaxomab) is possible. ${ }^{8,31}$

Recent studies report that free drainage of MA in a group of patients with EOC was safe and did not cause significant hypotension. ${ }^{18,48,49}$ There is no evidence to support the use of concomitant plasma expanders for hypotension prophylaxis. ${ }^{2,18}$ According to survey studies, intravenous fluids were always prescribed by $2 \%$ of physicians, and sometimes by $38 \%-42 \%$. When used, they were crystalloids (38\%-61\%), colloids $(11 \%)$, or albumin $(17 \%-40 \%) .{ }^{19,20}$

Intraperitoneal pressure (IAP) decreased significantly after drainage of 1000-1500 mL from ascites. With all the ascites drained, IAP was maintained at $11-12 \mathrm{~cm} \mathrm{H}_{2} \mathrm{O}$ and at $6-8 \mathrm{~cm} \mathrm{H}_{2} \mathrm{O}$ after $24 \mathrm{~h}$. Blood pressure was stable without significant changes before and after IAP reduction $(P>0.05)$. The breath rate and heart rate were improved, and $24 \mathrm{~h}$ urinary volume increased significantly after IAP reduction $(P<0.01) .{ }^{50}$

Historically, repeated PC has been associated with significant reduction in serum protein levels. However, the results of the cited studies do not support this statement. Moreover, there is a balance between albumin intake, metabolism, synthesis, and loss, which is multifactorial. ${ }^{51}$ The concentration of visceral proteins such as serum albumin is a good indicator of disease severity and outcome. It should not be used for either screening or diagnosis of malnutrition. ${ }^{52}$ Based on a meta-analysis of six randomized controlled trials comparing the administration of albumin with that of no albumin in cohorts of patients with ascites and liver cirrhosis, Vincent et al found a 0.72 risk ratio for morbidity in patients receiving albumin vs no albumin. ${ }^{53}$ However, there was not a single patient with MA in the evaluated trials.

\section{Conclusion}

Selection of the most appropriate MA drainage method for individual patients is crucial for successful management. However, this task is difficult. First, a careful interview with the patient, best with the usage of QOL questionnaires, should provide a list and type of symptoms and QOL issues that could be targeted by ascites drainage. Second, the underlying malignancy and treatment plans are important in terms of expected survival and perceived benefits or harms of MA drainage (eg, EOC vs others). Some patients will benefit from single PC, while others will be good candidates for TPC or PPP insertion and ascites drainage followed by chemotherapy. Drainage by CVC is an interesting method because the insertion is simple and does not involve many health care resources, while the patient can benefit from all 
the advantages of TPC. Special attention and experience are crucial for selecting patients for PVS because serious complications are to be expected. The PS of patients is an important factor. However, there are conflicting data as to whether those with poor PS will benefit from ascites drainage at all. The amount of ascitic fluid should not guide the decision and the goal of MA drainage is not drainage to dryness, but a symptom control and QOL improvement. Ascites drainage should be perceived as a supportive care that can be applied for those who need it at any time of their cancer trajectory, in order to better control symptoms, QOL, and treatment outcomes.

\section{Author contributions}

The author conceived and designed the study, performed the literature search, data selection, and interpretation, drafted the manuscript, provided final approval of the version to be published and agreed to be accountable for all aspects of the work.

\section{Disclosure}

The author reports no conflicts of interest in this work.

\section{References}

1. Orsi F, Grasso RF, Bonomo G, Monti C, Marinucci I, Bellomi M. Percutaneous peritoneovenous shunt positioning: technique and preliminary results. Eur Radiol. 2002;12(5):1188-1192.

2. Becker G, Galandi D, Blum HE. Malignant ascites: systematic review and guideline for treatment. Eur J Cancer. 2006;42(5):589-597.

3. Akinci D, Erol B, Ciftci TT, Akhan O. Radiologically placed tunneled peritoneal catheter in palliation of malignant ascites. Eur J Radiol. 2011;80(2):265-268

4. Coupe NA, Cox K, Clark K, Boyer M, Stockler M. Outcomes of permanent peritoneal ports for the management of recurrent malignant ascites. J Palliat Med. 2013;16(8):938-940.

5. Courtney A, Nemcek AA Jr, Rosenberg S, Tutton S, Darcy M, Gordon G. Prospective evaluation of the PleurX catheter when used to treat recurrent ascites associated with malignancy. J Vasc Interv Radiol. 2008;19(12):1723-1731.

6. Gough IR, Balderson GA. Malignant ascites. A comparison of peritoneovenous shunting and nonoperative management. Cancer. 1993;71(7):2377-2382.

7. Lungren MP, Kim CY, Stewart JK, Smith TP, Miller MJ. Tunneled peritoneal drainage catheter placement for refractory ascites: single-center experience in 188 patients. JVasc Interv Radiol. 2013;24(9):1303-1308.

8. Maleux G, Indesteege I, Laenen A, Verslype C, Vergote I, Prenen H. Tenckhoff tunneled peritoneal catheter placement in the palliative treatment of malignant ascites: technical results and overall clinical outcome. Radiol Oncol. 2016;50(2):197-203.

9. Rosenberg S, Courtney A, Nemcek AA Jr, Omary RA. Comparison of percutaneous management techniques for recurrent malignant ascites. J Vasc Interv Radiol. 2004;15(10):1129-1131.

10. Easson AM, Bezjak A, Ross S, Wright JG. The ability of existing questionnaires to measure symptom change after paracentesis for symptomatic ascites. Ann Surg Oncol. 2007;14(8):2348-2357.

11. Seike M, Maetani I, Sakai Y. Treatment of malignant ascites in patients with advanced cancer: peritoneovenous shunt versus paracentesis. J Gastroenterol Hepatol. 2007;22(12):2161-2166.
12. Parsons SL, Lang MW, Steele RJ. Malignant ascites: a 2-year review from a teaching hospital. Eur J Surg Oncol. 1996;22(3):237-239.

13. Kipps E, Tan DS, Kaye SB. Meeting the challenge of ascites in ovarian cancer: new avenues for therapy and research. Nat Rev Cancer. 2013;13(4):273-282.

14. Fleming ND, Alvarez-Secord A, Von Gruenigen V, Miller MJ, Abernethy AP. Indwelling catheters for the management of refractory malignant ascites: a systematic literature overview and retrospective chart review. J Pain Symptom Manage. 2009;38(3):341-349.

15. Pockros PJ, Esrason KT, Nguyen C, Duque J, Woods S. Mobilization of malignant ascites with diuretics is dependent on ascitic fluid characteristics. Gastroenterology. 1992;103(4):1302-1306.

16. Keen A, Fitzgerald D, Bryant A, Dickinson HO. Management of drainage for malignant ascites in gynaecological cancer. Cochrane Database Syst Rev. 2010(1):CD007794.

17. Gamblin V, Da Silva A, Villet S, El Hajbi F. [Supportive care for malignant ascites in palliative phase: place of paracentesis and diuretics]. Bull Cancer. 2015;102(11):940-945. French.

18. Harding V, Fenu E, Medani H, et al. Safety, cost-effectiveness and feasibility of daycase paracentesis in the management of malignant ascites with a focus on ovarian cancer. Br J Cancer. 2012;107(6):925-930.

19. Newman G, Pudney D. A survey of current practice in the management of recurrent malignant ascites among oncologists and palliative-care physicians in the UK. Clin Oncol (R Coll Radiol). 2006;18(2):154.

20. Jehn CF, Kupferling S, Oskay-Ozcelik G, Luftner D. A survey of treatment approaches of malignant ascites in Germany and Austria. Support Care Cancer. 2015;23(7):2073-2078.

21. Lee CW, Bociek G, Faught W. A survey of practice in management of malignant ascites. J Pain Symptom Manage. 1998;16(2):96-101.

22. Shamseer L, Moher D, Clarke M, et al. Preferred reporting items for systematic review and meta-analysis protocols (PRISMA-P) 2015: elaboration and explanation. BMJ. 2015;349:g7647.

23. White J, Carolan-Rees G. PleurX peritoneal catheter drainage system for vacuum-assisted drainage of treatment-resistant, recurrent malignant ascites: a NICE Medical Technology Guidance. Appl Health Econ Health Policy. 2012;10(5):299-308.

24. Deshpande PR, Rajan S, Sudeepthi BL, Abdul Nazir CP. Patientreported outcomes: a new era in clinical research. Perspect Clin Res. 2011;2(4):137-144.

25. Tapping CR, Ling L, Razack A. PleurX drain use in the management of malignant ascites: safety, complications, long-term patency and factors predictive of success. Br J Radiol. 2012;85(1013):623-628.

26. Mercadante S, Intravaia G, Ferrera P, Villari P, David F. Peritoneal catheter for continuous drainage of ascites in advanced cancer patients. Support Care Cancer. 2008;16(8):975-978.

27. Stukan M, Lesniewski-Kmak K, Wroblewska M, Dudziak M. Management of symptomatic ascites and post-operative lymphocysts with an easy-to-use, patient-controlled, vascular catheter. Gynecol Oncol. 2015;136(3):466-471.

28. Gu X, Zhang Y, Cheng M, Liu M, Zhang Z, Cheng W. Management of non-ovarian cancer malignant ascites through indwelling catheter drainage. BMC Palliat Care. 2016;15:44.

29. Monsky WL, Yoneda KY, MacMillan J, et al. Peritoneal and pleural ports for management of refractory ascites and pleural effusions: assessment of impact on patient quality of life and hospice/home nursing care. J Palliat Med. 2009;12(9):811-817.

30. Ghaffar MKA, Hassan MS, Mostafa MY. Value of implantable peritoneal ports in managing recurrent malignant ascites. Egypt J Radiol Nucl Med. 2014;45(2):417-422.

31. Ozkan O, Akinci D, Gocmen R, Cil B, Ozmen M, Akhan O. Percutaneous placement of peritoneal port-catheter in patients with malignant ascites. Cardiovasc Intervent Radiol. 2007;30(2):232-236.

32. Savin MA, Kirsch MJ, Romano WJ, Wang SK, Arpasi PJ, Mazon CD. Peritoneal ports for treatment of intractable ascites. JVasc Interv Radiol. 2005;16(3):363-368.

33. Richard HM 3rd, Coldwell DM, Boyd-Kranis RL, Murthy R, Van Echo DA. Pleurx tunneled catheter in the management of malignant ascites. J Vasc Interv Radiol. 2001;12(3):373-375. 
34. Belfort MA, Stevens PJ, DeHaek K, Soeters R, Krige JE. A new approach to the management of malignant ascites; a permanently implanted abdominal drain. Eur J Surg Oncol. 1990;16(1):47-53.

35. Zanon C, Grosso M, Apra F, et al. Palliative treatment of malignant refractory ascites by positioning of Denver peritoneovenous shunt. Tumori. 2002;88(2):123-127.

36. Hussain FF, Meer ZF, Lopez AJ. Peritoneovenous shunt insertion for intractable ascites: a district general hospital experience. Cardiovasc Intervent Radiol. 2004;27(4):325-328.

37. Bratby MJ, Hussain FF, Lopez AJ. Radiological insertion and management of peritoneovenous shunt. Cardiovasc Intervent Radiol. 2007;30(3):415-418.

38. Macdonald S, Watt AJ, McNally D, Edwards RD, Moss JG. Comparison of technical success and outcome of tunneled catheters inserted via the jugular and subclavian approaches. J Vasc Interv Radiol. 2000; 11(2 Pt 1):225-231.

39. Soderlund C. Denver peritoneovenous shunting for malignant or cirrhotic ascites. A prospective consecutive series. Scand J Gastroenterol. 1986;21(10):1161-1172.

40. Tomiyama K, Takahashi M, Fujii T, et al. Improved quality of life for malignant ascites patients by Denver peritoneovenous shunts. Anticancer Res. 2006;26(3B):2393-2395.

41. Husain A, Bezjak A, Easson A. Malignant ascites symptom cluster in patients referred for paracentesis. Ann Surg Oncol. 2010;17(2): 461-469.

42. Ross GJ, Kessler HB, Clair MR, Gatenby RA, Hartz WH, Ross LV. Sonographically guided paracentesis for palliation of symptomatic malignant ascites. AJR Am J Roentgenol. 1989;153(6):1309-1311.

43. Barnett TD, Rubins J. Placement of a permanent tunneled peritoneal drainage catheter for palliation of malignant ascites: a simplified percutaneous approach. J Vasc Interv Radiol. 2002;13(4):379-383.

44. Ayantunde AA, Parsons SL. Pattern and prognostic factors in patients with malignant ascites: a retrospective study. Ann Oncol. 2007;18(5):945-949.
45. Hui D, Bruera E. Integrating palliative care into the trajectory of cancer care. Nat Rev Clin Oncol. 2016;13(3):159-171.

46. ElJawahri A, Jackson VA, Greer JA, et al. Early integrated palliative care to improve family caregivers (FC) outcomes for patients with gastrointestinal and lung cancer. J Clin Oncol. 2016;34(Suppl):abstr 10131.

47. Zamboni CG, Azene EM, Higgins LJ, Hong K. Does chemotherapy increase infection rates in cancer patients with an indwelling PleurX ${ }^{\circledR}$ abdominal drainage catheter for malignant ascites? J Vasc Intervent Radiol. 2014;25(3 Suppl):S126.

48. Decruze SB, Macdonald R, Smith G, Herod JJ. Paracentesis in ovarian cancer: a study of the physiology during free drainage of ascites. J Palliat Med. 2010;13(3):251-254.

49. Gotlieb WH, Feldman B, Feldman-Moran O, et al. Intraperitoneal pressures and clinical parameters of total paracentesis for palliation of symptomatic ascites in ovarian cancer. Gynecol Oncol. 1998;71(3):381-385.

50. Wang HY, An F, Yang XY, Yang XF, Ran XG. [Clinical outcome after pressure reduction by peritoneal catheterization in 29 patients with malignant ascites-induced abdominal compartment syndrome]. Zhonghua Wei Chang Wai Ke Za Zhi. 2010;13(4):273-275. Chinese.

51. Franch-Arcas $\mathrm{G}$. The meaning of hypoalbuminaemia in clinical practice. Clin Nutr. 2001;20(3):265-269.

52. Cederholm T, Bosaeus I, Barazzoni R, et al. Diagnostic criteria for malnutrition - an ESPEN consensus statement. Clin Nutr. 2015;34(3):335-340.

53. Vincent JL, Navickis RJ, Wilkes MM. Morbidity in hospitalized patients receiving human albumin: a meta-analysis of randomized, controlled trials. Crit Care Med. 2004;32(10):2029-2038.

54. Narayanan G, Pezeshkmehr A, Venkat S, Guerrero G, Barbery K. Safety and efficacy of the PleurX catheter for the treatment of malignant ascites. $J$ Palliat Med. 2014;17(8):906-912.

55. O’Neill MJ, Weissleder R, Gervais DA, Hahn PF, Mueller PR. Tunneled peritoneal catheter placement under sonographic and fluoroscopic guidance in the palliative treatment of malignant ascites. AJR Am J Roentgenol. 2001;177(3):615-618.
Cancer Management and Research

\section{Publish your work in this journal}

Cancer Management and Research is an international, peer-reviewed open access journal focusing on cancer research and the optimal use of preventative and integrated treatment interventions to achieve improved outcomes, enhanced survival and quality of life for the cancer patient. The manuscript management system is completely online and includes

\section{Dovepress}

a very quick and fair peer-review system, which is all easy to use. Visit http://www.dovepress.com/testimonials.php to read real quotes from published authors. 